\title{
Robotic Radical Partial Nephrectomy: Keep Pushing the Boundaries
}

\author{
*Sanchia Goonewardene \\ The Royal Free and UCL, UK
}

Submission: October 26, 2016; Published: October 18, 2016

"Corresponding author: Sanchia Goonewardene, The Royal Free and UCL, NW3 2NG London, UK

\begin{abstract}
The purpose of robotic radical partial nephrectomy is to ensure oncological clearance, whilst maintaining renal function. Main renal artery clamping during robotic partial nephrectomy (RPN) may compromise post-operative renal function [1]. Recent technologic advances mean resectional technique and outcomes can be improved. Specifically, robotic platforms, with robotic-assisted instrumentation, have helped overcome previous barriers to widespread adoption of laparoscopic surgery [2]. Image guidance can improve surgeon ease, accuracy, and comfort with complex procedures [2]. How can urologists make use of these advances to improve oncological and renal function outcomes during and after robotic radical partial nephrectomy?
\end{abstract}

Keywords: Robotic radical Nephrectomy; Resection; Fluorescence

Abbreviations: RPN: Robotic Partial Nephrectomy; NIR: Near Infrared; ICG: Indocyanine Green; NIRF: Near-Infrared Fluorescence; ICG: Indocyanine Green

\section{Mini Review}

Modern technologies such as near infrared (NIR) indocyanine green (ICG) fluorescence imaging represent potentially useful tools to facilitate intraoperative assessment of vascularization and tissue perfusion [3]. Recently, the use of a near-infrared fluorescence (NIRF) imaging system using indocyanine green (ICG) dye has been described during robotic partial nephrectomy as an adjunctive means of identifying renal vasculature and tumour clearance. ICG is a water-soluble dye that fluoresces bright green when viewed under near-infrared light (700-1000 $\mathrm{nm}$ ) [4]. This technology has been applied to robotic partial nephrectomy, to look for the differentiation of renal tumor from normal parenchyma [4]. In this application, it has been hypothesized that normal kidney tissue fluoresces green, while the tumor commonly remains hypofluorescent, thereby aiding tumor excision [4]. Secondly, NIRF imaging with ICG has been employed to facilitate selective arterial clamping during robotic partial nephrectomy, allowing for a regional perfusion deficit in the kidney to be readily identified and therefore targeted at a given tumor [4].

The technique described is one of a standard lateral position with transperitoneal approach [5]. Two $8 \mathrm{~mm}$ robotic working ports were triangulated with the robotic camera port. $5 \mathrm{~mm}$ and $10 \mathrm{~mm}$ assistant ports are usually used. The main renal artery and segmental renal arteries would be isolated [5]. The renal tumour can then be exposed by opening a window in Gerota's fascia [5]. With the aid of intraoperative ultrasound, the margins of the tumour can be identified, and the planned resection margin scored with diathermy. A bulldog vascular clamp was applied to selected renal arteries, followed immediately by administration of $2 \mathrm{ml}$ (5 mg) of intravenous ICG [5]. NIRF demonstrated no fluorescence, meaning, selective ischaemia of the selected zone [5]. The tumour showed no fluorescence compared to the normal renal parenchyma [5]. Excision can be performed by switching between white light and fluorescence modes [5].

A layer of green fluorescing normal renal parenchyma covering the base of the resected specimen provided intraoperative reassurance of a negative margin. NIRF using intravenously injected ICG has emerged as a useful adjunct to robotic partial nephrectomy. It provides the surgeon with a visual contrast between normal parenchyma and the tumour, and allows demonstration of regional perfusion deficit after segmental clamping of renal vasculature, facilitating a bloodless surgical field [5]. Additionally this tool can also be used to check the perfusion of renal parenchyma was also checked with ICG fluorescence after completion of the renorrhaphy [3]. Intraoperative NIR ICG fluorescence imaging represents a useful tool to support surgical decisions during RAPN. This can give good results [3]. This study used a test of selective clamping of 


\section{Cancer Therapy \& Oncology International Journal}

the tumour-feeding arteries after injection of $10 \mathrm{mg}$ of ICG to assess the adequacy of tumor ischemia [3].

The perfusion of renal parenchyma was checked with ICG fluorescence after completion of the renorrhaphy [3]. This demonstrated intraoperative NIR ICG fluorescence imaging represents a useful tool to support surgical decisions during RAPN [3]. Additionally, recent studies have shown the associated decrease in global ischemia to minimize resultant loss of renal function at certain time endpoints [4]. This system can also be used to confirm the definite border between normal and tumor kidney tissues [6].ICG-based systems have been helpful for confirming negative margin status in even the most complex cases [6]. Intraoperative imaging of ICG with NIRF is a safe and effective method to accurately identify the renal vasculature and to differentiate renal tumors from surrounding normal parenchyma [7]. The capacity for multimodal imaging within the surgical console further facilitates this imaging [7].

Due to the growing possibilities especially in robot-assisted partial nephrectomy (RPN) even large, hilar or intrarenal tumours can be removed [8]. Nevertheless a short ischemia time should be a major goal also in complex tumours [9]. This can be achieved using super-selective clamping of the tumour feeding vessels based on fluorescence imaging [9]. Robotic partial nephrectomy with selective clamping of specific tumour feeding arterial branches using indocyanine green can be performed safely even in complex tumour constellations [9]. The minimized ischemic trauma to the remaining parenchyma may lead to superior renal function preservation and significant reduced eGFR decrease.

However, this method should be used with caution for visualization of renal vasculature that is not at the surface of the kidney or complex anatomy [10]. Robotic partial nephrectomy with selective arterial clamping, when used with nearinfrared fluorescence imaging and indocyanine green, can be a safe approach to resecting renal masses if the patient's vascular anatomy is favourable [10]. A major drawback is that those vessels not at the surface of the kidney cannot be visualized with this technique and may result in excess bleeding during tumor excision [10]. Tumour complexity and perioperative variables are not associated with successful selective clamping, which may be related to complexity of renal vascular anatomy [11]. Further studies are warranted to identify anatomic characteristics associated with successful arterial clamping [12].

Its use has also been examined amongst various histologic subtypes [12]. This technique is safe and effective during robotic partial nephrectomy of various histologic subtypes [12]. Indocyanine Green (ICG) is emerging as a potential adjunct to robotic partial nephrectomy by its ability to help in the realtime identification of renal vasculature and the neoplasmparenchymal margin [13]. ICG dye does not appear to significantly alter distance of renal margin [13]. The mild increase in distance is likely attributable to ensuring equal fluorescent appearance on both sides of the resected tissue [13]. Facilitation of tumor excision may require proper dosing of indocyanine green [14]. Under dosing causes inadequate fluorescence of adjacent tumour parenchyma [14]. Overdosing causes tumours to fluoresce inappropriately. Two doses of indocyanine green have proven highly reliable in achieving differential fluorescence of kidney and renal cell carcinomas [14]. However, important points to note are the fluorescence patterns of renal masses have not been adequately described according to histology, and it remains unknown if fluorescence pattern can reliably predict histology or malignancy [14]. A three-grade classification of renal mass ICG fluorescence pattern is correlated with some histologic findings but unable to reliably predict malignant vs benign lesions [15].

This is especially important to consider when considering staged bilateral robotic partial nephrectomies are a safe and feasible method of managing bilateral renal masses [16]. An approach involving the initial management of the kidney at highest-risk of becoming a solitary kidney can successfully control malignancy while preventing progression to dialysis [16]. In conclusion, near infrared imaging using fluorescence properties of indocyanine green appears useful for identification of arterial branches that can be clamped selectively and in order to reduce the amount of renal parenchyma exposed to warm ischemia. Very short temporary test-clamping of various branches can also be performed to identify the correct supplying vessel before partial nephrectomy. With this technique, the majority of key steps from the hands of the first assistant and utilizes current immunofluorescence technology to allow for safe selective arterial clamping for amenable tumours. Special equipment for NIR imaging (light source, camera with filter and NIR optimized scope) is necessary.

\section{References}

1. Borofsky MS, Gill IS, Hemal AK, Marien TP, Jayaratna I, et al. (2013) Near infra-red fluorescence imaging to facilitate super-selective arterial clamping during zero-ischemia robotic partial nephrectomy. BJU Int 111(4): 604-610

2. Mitchell CR, Herrell SD (2014) Image-Guided Surgery and Emerging Molecular Imaging: Advances to Complement Minimally Invasive Surgery. Urol Clin North Am 41(4): 567-580.

3. Gan M, Volpe A, Ficarra V, De Naeyer G, Stifelman M, et al. (2013) Application of near-infrared fluorescence imaging in robot-assisted surgery. Journal of Endourology 27: A101.

4. Bjurlin MA, McClintock TR, Stifelman MD (2015) Near-Infrared Fluorescence Imaging with Intraoperative Administration of Indocyanine Green for Robotic Partial Nephrectomy. Curr Urol Rep 16(4): 20.

5. Yao J, McLeod N, Lau H (2014) Indocyanine green-based fluorescence imaging: Application during robotic partial nephrectomy. BJU Int 113: 97.

6. Mitsui Y, Shiina H, Arichi N, Hiraoka T, Inoue S, et al. (2012) Indocyanine green (ICG)-based fluorescence navigation system for discrimination of kidney cancer from normal parenchyma: Application during partial nephrectomy. Int Urol Nephrol 44(3): 753-759. 


\section{Cancer Therapy \& Oncology International Journal}

7. Tobis S, Knopf J, Silvers C, Yao J, Rashid H, et al. (2011) Near infrared fluorescence imaging for the da Vinci Si Surgical system: Initial clinical experience with robot-assisted laparoscopic partial nephrectomy for renal cortical tumors. J Urol 186(1): 47-52.

8. Harke NN, Schiefelbein F, Schoen G (2014) Improved short-term renal function after robot-assisted partial nephrectomy with selective arterial clamping-a matched pair analysis for preoperative GFR European Urology 13(3): 4-5

9. Harke NN, Schiefelbein F, Schoen G (2015) Superior renal function after selective clamping robotic partial nephrectomy-a matched pair study for baseline eGFR with short-and intermediate-term follow-up. European Urology, Supplements 14 (2): e920.

10. Bahler CD, Shatagopam K, Sundaram CP (2014) Utility of indocyanine green in robotic partial nephrectomy with selective arterial clamping. J Endourol 28: A286.

11. Rothberg MB, Weinberg AC, Korets R, Badani KK (2014) Utilization and success rate of selective arterial clamping using immunofluoresence imaging during robotic partial nephrectomy. Journal of Endourology 28: A30

12. Manny TB, Krane LS, Hemal AK (2013) Indocyanine green cannot predict malignancy in robotic partial nephrectomy: Histopathologic correlation in 100 patients. J Endourol 27(7): 918-921.

13. Krane LS, Manny T, Manny JS, Hemal AK (2012) Indocyanine green in partial nephrectomy: Impact on surgical margin distance. J Endourol 26: A250.

14. Angell JE, Khemees TA, Abaza R (2013) Optimization of near infrared fluorescence tumor localization during robotic partial nephrectomy. J Urol 190(5): 1668-1673.

15. Manny T, Krane S, Richards K, Hemal A (2012) Indocyanine green cannot predict histology or malignancy: Fluorescence patterns in a variety of renal masses. BJU Int 110: 64-65.

16. Ted Manny, Manish Patel, Ashok Hemal (2013) Robotic partial nephrectomy for bilateral tumors: Secisions, techniques, \& outcomes. Journal of Urology 189(4): e444. 